\title{
A New Approach to Improve Accuracy of Grey Model GMC $(1, n)$ in Time Series Prediction
}

\author{
Sompop Moonchai ${ }^{1}$ and Wanwisa Rakpuang ${ }^{2}$ \\ ${ }^{1}$ Department of Mathematics, Faculty of Science, Chiang Mai University, Chiang Mai 50200, Thailand \\ ${ }^{2}$ Department of Mathematics, Faculty of Science and Technology, Kamphaeng Phet Rajabhat University, \\ Kamphaeng Phet 62000, Thailand \\ Correspondence should be addressed to Sompop Moonchai; tumath@gmail.com
}

Received 2 September 2015; Accepted 17 November 2015

Academic Editor: Min-Chie Chiu

Copyright (C) 2015 S. Moonchai and W. Rakpuang. This is an open access article distributed under the Creative Commons Attribution License, which permits unrestricted use, distribution, and reproduction in any medium, provided the original work is properly cited.

\begin{abstract}
This paper presents a modified grey model $\operatorname{GMC}(1, n)$ for use in systems that involve one dependent system behavior and $n-1$ relative factors. The proposed model was developed from the conventional $\operatorname{GMC}(1, n)$ model in order to improve its prediction accuracy by modifying the formula for calculating the background value, the system of parameter estimation, and the model prediction equation. The modified $\operatorname{GMC}(1, n)$ model was verified by two cases: the study of forecasting $\mathrm{CO}_{2}$ emission in Thailand and forecasting electricity consumption in Thailand. The results demonstrated that the modified GMC $(1, n)$ model was able to achieve higher fitting and prediction accuracy compared with the conventional GMC $(1, n)$ and D-GMC $(1, n)$ models.
\end{abstract}

\section{Introduction}

Grey model is a useful tool for modeling and forecasting future values of a system based on the information and knowledge obtained from the past and current data. Grey model was developed from the grey system theory introduced by Deng in the early 1980s [1]. It can be used to predict behaviors of systems in the future value with high accuracy without knowing their mathematical models and used in the uncertain coefficients system with small nonnegative data. Grey model has been successfully applied to various systems $[1-8] . \operatorname{GM}(m, n)$ denotes a grey model which indicates that $n$ variables are employed in the model and that it is an $m$ order differential equation. $\operatorname{GM}(1,1)$ is a first-order onevariable grey differential equation, and it is the most widely used grey model in time series prediction [9]. However, it does not find application in multivariable prediction models which are important for real applied works [4, 5, 10-13]. The grey prediction models have been expanded from the original $\operatorname{GM}(1,1)$ to novel prediction types, such as $\operatorname{GM}(1, n)$ [14], $\operatorname{GMC}(1, n)[10,15], \mathrm{D}-\operatorname{GMC}(1, n)[14], \operatorname{DGDMC}(1, n)$ [16], and $\operatorname{CAGM}(1, n)[17] . \operatorname{GM}(1, n)$ model is a grey multivariable model used for estimating the relationship between the system behavior and $n-1$ relative factors [14] and has been used for various applications [4, 11, 18-20]. However, there are some limitations existing in the $\operatorname{GM}(1, n)$ which affect the prediction accuracy of $\operatorname{GM}(1, n)[14,15]$. Then, the grey multivariable model with the convolution integral $\operatorname{GMC}(1, n)$, proposed by Tien [10], is developed from $\operatorname{GM}(1, n)$ by adding the grey control parameter in the differential equation of $\operatorname{GM}(1, n)$ to improve the forecasting accuracy of $\operatorname{GM}(1, n)$. The $\operatorname{GMC}(1, n)$ model has successfully been applied to many real works $[10,15,21,22]$. However, the prediction accuracy of the $\operatorname{GMC}(1, n)$ model depends on many factors such as smooth condition of the raw data, background value calculation, and model prediction equation. Moreover, there exists a contradiction between discrete equations for parameter estimation and continuous equations for model predictions $[5,23]$. Therefore, a high accuracy of prediction cannot be expected of $\operatorname{GMC}(1, n)$ for an actual system $[8,16]$.

In this paper, we proposed a modified grey $\operatorname{GMC}(1, n)$ model to improve the prediction accuracy of the conventional $\operatorname{GMC}(1, n)$ by modification of the formula for calculating the background value, the system of the parameter estimation, and the model prediction equation. In addition, we presented the case studies with the numerical results for prediction 
accuracy for the modified GMC $(1, n)$ model in comparison with the conventional $\operatorname{GMC}(1, n)$ and the discrete multivariate grey model D-GMC $(1, n)[5]$.

The paper is organized as follows: Section 2 describes the conventional GMC $(1, n)$, Section 3 proposes a modified grey GMC $(1, n)$ model, Section 4 explains the statistical measure of the forecasting performance, and Section 5 presents the case study with the modified grey $\operatorname{GMC}(1, n)$. Finally, the conclusions are drawn in Section 6.

\section{2. $\mathbf{G M C}(1, n)$ Model $[8,10]$}

Suppose that the original multivariate time series $X_{i}^{(0)}=$ $\left\{X_{i}^{(0)}(1), X_{i}^{(0)}(2), \ldots, X_{i}^{(0)}(r)\right\}, i=1,2, \ldots, n$ are a nonnegative series and available at an equispaced interval of time, where the main factor of the system behavior is $X_{1}^{(0)}$, the relative factors are $X_{i}^{(0)}, i=2,3, \ldots, n$, and $r$ is the number of data. Then the first-order accumulative generation operation (1-AGO) of $X_{i}^{(0)}, i=1,2, \ldots, n$, is given by the following equation:

$$
X_{i}^{(1)}=\left\{X_{i}^{(1)}(1), X_{i}^{(1)}(2), \ldots, X_{i}^{(1)}(r)\right\},
$$

$$
i=1,2, \ldots, n \text {, }
$$

where $X_{i}^{(1)}(k)=\sum_{j=1}^{k} X_{i}^{(0)}(j), i=1,2, \ldots, n, k=1,2, \ldots, r$.

The prediction procedure of using the conventional $\operatorname{GMC}(1, n)$ model is shown as follows.

The grey prediction model based on the 1-AGO data, $X_{i}^{(1)}, i=1,2, \ldots, n$, is given by the following differential equation:

$$
\begin{aligned}
\frac{d X_{1}^{(1)}(t)}{d t}+b_{1} X_{1}^{(1)}(t)= & b_{2} X_{2}^{(1)}(t)+b_{3} X_{3}^{(1)}(t)+\cdots \\
& +b_{n} X_{n}^{(1)}(t)+u \\
& t=1,2, \ldots, r f
\end{aligned}
$$

where $b_{1}, b_{2}, \ldots, b_{n}$ and $u$ are model parameters to be estimated and $r f$ is the number of entries to be predicted.

Taking the integral of both sides of (2) in the interval [ $k-$ $1, k]$ yields

$$
\begin{aligned}
X_{1}^{(0)}(k)+b_{1} z_{1}^{(1)}(k)= & b_{2} z_{2}^{(1)}(k)+b_{3} z_{3}^{(1)}(k)+\cdots \\
& +b_{n} z_{n}^{(1)}(k)+u
\end{aligned}
$$

where $z_{i}^{(1)}(k)=\int_{k-1}^{k} X_{i}^{(1)}(t) d t$ for $i=1,2, \ldots, n$, which are called the background values [9].

By using the trapezoidal rule, the values of $\int_{k-1}^{k} X_{i}^{(1)}(t) d t$ for $i=1,2, \ldots, n$ are approximated by the following equation:

$$
z_{i}^{(1)}(k) \approx \frac{X_{i}^{(1)}(k)+X_{i}^{(1)}(k-1)}{2} t, \quad i=1,2, \ldots, n .
$$

Substituting all the data values in (3) gives the system of linear equations that can be written as a matrix equation in the form

$$
A b=Y \text {, }
$$

where

$$
\begin{aligned}
& A=\left[\begin{array}{cccccc}
-z_{1}^{(1)}(2) & z_{2}^{(1)}(2) & \cdots & z_{n}^{(1)}(2) & 1 \\
-z_{1}^{(1)}(3) & z_{2}^{(1)}(3) & \cdots & z_{n}^{(1)}(3) & 1 \\
\vdots & \vdots & \ddots & \vdots & \vdots \\
-z_{1}^{(1)}(r) & z_{2}^{(1)}(r) & \cdots & z_{n}^{(1)}(r) & 1
\end{array}\right] \\
& Y=\left[\begin{array}{c}
X_{1}^{(0)}(2) \\
X_{1}^{(0)}(3) \\
\vdots \\
X_{1}^{(0)}(r)
\end{array}\right] \\
& b=\left[\begin{array}{llllll}
b_{1} & b_{2} & b_{3} & \cdots & b_{n} & u
\end{array}\right]^{T} \text {. }
\end{aligned}
$$
obtain

Applying the least square method to this system, we

$$
b=\left(A^{T} A\right)^{-1} A^{T} Y
$$

Solving (2) with the initial condition $\widehat{X}_{1}^{(1)}(1)=X_{1}^{(1)}(1)$, the predicted 1-AGO series is obtained as follows:

$$
\begin{aligned}
& \widehat{X}_{1}^{(1)}(k+1) \\
& =X_{1}^{(0)}(1) e^{-b_{1} k} \\
& \quad+\theta(k-1) \sum_{i=2}^{k+1}\left[e^{-b_{1}(k+3 / 2-i)} \frac{1}{2}(f(i)+f(i-1))\right], \\
& f(i)=b_{2} X_{2}^{(1)}(i)+b_{3} X_{3}^{(1)}(i)+\cdots+b_{n} X_{n}^{(1)}(i)+u,
\end{aligned}
$$

where $\theta(k-1)$ is the unit step function and $k=2,3, \ldots, r f$ and $\widehat{X}_{1}^{(1)}(k)$ denotes the predicted value of $X_{1}^{(1)}(k)$.

Therefore, the predict series of $X_{1}^{(0)}(k), k=2,3, \ldots, r f$ is given by

$$
\widehat{X}_{1}^{(0)}(k)=\widehat{X}_{1}^{(1)}(k)-\widehat{X}_{1}^{(1)}(k-1) .
$$

\section{Modified GMC $(1, n)$ Model}

The proposed model is modified from $\operatorname{GMC}(1, n)$ by using two advanced improvements, as follows:

(1) The equation of the predicted 1-AGO series is obtained accurately from the exactly integrated form of $X_{i}^{(1)}$.

(2) The system of parameter estimation is derived by using the model prediction equation in order to eliminate the problem of a contradiction between the system of discrete equations for parameter estimation and the system of continuous equations for model predictions.

The grey model can perform well as regards predictions if the raw data satisfies the quasi-smooth and quasi-exponential conditions [17, 24]. However, if the raw data does not satisfy these conditions, function transformation methods are applied in original data sequences [17]. Suppose that 
the original data $X_{i}^{(0)}, i=1,2, \ldots, n$, satisfy the quasismooth and quasi-exponential condition. Then, the 1-AGO data $X_{i}^{(1)}, i=1,2,3, \ldots, n$, are fitted by the exponential function $[9,17]$, which can be written as

$$
\begin{aligned}
X_{i}^{(1)}(k)=E_{i} e^{a_{i} k}+F_{i}, & \\
& i=1,2,3, \ldots, n, k=1,2,3, \ldots, r .
\end{aligned}
$$

Next, parameters $E_{i}, a_{i}$, and $F_{i}$ are determined using the least square method, which is as follows:

From (9), the raw data can be expressed by the 1-AGO data as

$$
\begin{aligned}
X_{i}^{(0)}(k-1) & =X_{i}^{(1)}(k-1)-X_{i}^{(1)}(k-2) \\
& =E_{i} e^{a_{i}(k-2)}\left(e^{a_{i}}-1\right), \\
X_{i}^{(0)}(k) & =X_{i}^{(1)}(k)-X_{i}^{(1)}(k-1) \\
& =E_{i} e^{a_{i}(k-1)}\left(e^{a_{i}}-1\right) .
\end{aligned}
$$

Dividing (12) by (11), we have

$$
\begin{aligned}
X_{i}^{(0)}(k)=e^{a_{i}} X_{i}^{(0)}(k-1)=M_{i} X_{i}^{(0)}(k-1), & \\
& \text { where } M_{i}=e^{a_{i}} .
\end{aligned}
$$

Let $H\left(M_{i}\right)=\sum_{k=1}^{r}(1 / 2)\left(X_{i}^{(0)}(k)-M_{i} X_{i}^{(0)}(k-1)\right)^{2}$ be the objective function. By using the least square method, $H$ can be made minimum using the parameter $M_{i}$, which should satisfy

$$
\begin{aligned}
\frac{\partial H}{\partial M_{i}} & =-\sum_{k=1}^{r}\left(X_{i}^{(0)}(k)-M_{i} X_{i}^{(0)}(k-1)\right) X_{i}^{(0)}(k-1) \\
& =0 .
\end{aligned}
$$

By solving this equation, we can obtain

$$
\begin{gathered}
M_{i}=e^{a_{i}}=\frac{\sum_{k=1}^{r} X_{i}^{(0)}(k) X_{i}^{(0)}(k-1)}{\sum_{k=1}^{r}\left(X_{i}^{(0)}(k-1)\right)^{2}}, \\
a_{i}=\ln \left(\frac{\sum_{k=1}^{r} X_{i}^{(0)}(k) X_{i}^{(0)}(k-1)}{\sum_{k=1}^{r}\left(X_{i}^{(0)}(k-1)\right)^{2}}\right) .
\end{gathered}
$$

Substituting (15) into (10) gives

$$
X_{i}^{(1)}(k)=E_{i} W_{i}(k)+F_{i},
$$

where $W_{i}(k)=M_{i}^{k}=e^{a_{i} k}$.

Let $Q\left(E_{i}, F_{i}\right)=\sum_{k=1}^{r}(1 / 2)\left(X_{i}^{(1)}(k)-E_{i} W_{i}(k)-F_{i}\right)^{2}$ be the objective function. The $E_{i}$ and $F_{i}$ should satisfy

$$
\begin{aligned}
& \frac{\partial Q}{\partial E_{i}}=-\sum_{k=1}^{r}\left(X_{i}^{(1)}(k)-E_{i} W_{i}(k)-F_{i}\right) W_{i}(k)=0, \\
& \frac{\partial Q}{\partial F_{i}}=-\sum_{k=1}^{r}\left(X_{i}^{(1)}(k)-E_{i} W_{i}(k)-F_{i}\right)=0 .
\end{aligned}
$$

Solving this equation yields

$$
\begin{aligned}
E_{i} & \\
= & \frac{r \sum_{k=1}^{r} W_{i}(k) X_{i}^{(1)}(k)-\sum_{k=1}^{r} W_{i}(k) \sum_{k=1}^{r} X_{i}^{(1)}(k)}{r \sum_{k=1}^{r}\left(W_{i}(k)\right)^{2}-\left(\sum_{k=1}^{r} W_{i}(k)\right)^{2}} \\
& =\frac{r \sum_{k=1}^{r} e^{a_{i} k} X_{i}^{(1)}(k)-\sum_{k=1}^{r} e^{a_{i} k} \sum_{k=1}^{r} X_{i}^{(1)}(k)}{r \sum_{k=1}^{r}\left(e^{a_{i} k}\right)^{2}-\left(\sum_{k=1}^{r} e^{a_{i} k}\right)^{2}}, \\
F_{i} & =\frac{\sum_{k=1}^{r} X_{i}^{(1)}(k)}{r}-E_{i} \frac{\sum_{k=1}^{r} e^{a_{i} k}}{r} .
\end{aligned}
$$

From (10), the background value $z_{i}^{(1)}(k)$ can be written as follows:

$$
\begin{aligned}
z_{i}^{(1)}(k) & =\int_{k-1}^{k} X_{i}^{(1)}(t) d t=\int_{k-1}^{k}\left(E_{i} e^{a_{i} t}+F_{i}\right) d t \\
& =\left[\frac{E_{i} e^{a_{i} t}}{a_{i}}+F_{i} t\right]_{k-1}^{k} \\
& =\frac{E_{i} e^{a_{i} k}}{a_{i}}-\frac{E_{i} e^{a_{i}(k-1)}}{a_{i}}+F_{i}(k-(k-1)) \\
& =\frac{1}{a_{i}}\left(E_{i} e^{a_{i}(k)}-E_{i} e^{a_{i}(k-1)}\right)+F_{i}(k-(k-1)) \\
& =\frac{1}{a_{i}}\left(X_{i}^{(1)}(k)-X_{i}^{(1)}(k-1)\right)+F_{i} \\
& =\frac{X_{i}^{(0)}(k)}{a_{i}}+F_{i}, \quad i=1,2,3, \ldots, n .
\end{aligned}
$$

Substituting (20) into (3), we have

$$
\begin{aligned}
& X_{1}^{(0)}(k)+b_{1}\left(\frac{X_{1}^{(0)}(k)}{a_{1}}+F_{1}\right) \\
& =\sum_{i=2}^{n} b_{i}\left(\frac{X_{i}^{(0)}(k)}{a_{i}}+F_{i}\right)+u, \\
& \left(\frac{a_{1}+b_{1}}{a_{1}}\right) X_{1}^{(0)}(k) \\
& =\sum_{i=2}^{n} b_{i}\left(\frac{X_{i}^{(0)}(k)}{a_{i}}+F_{i}\right)+u-b_{1} F_{1},
\end{aligned}
$$

$X_{1}^{(0)}(k)$

$$
\begin{gathered}
=\sum_{i=2}^{n}\left(\frac{a_{1} b_{i}}{a_{1}+b_{1}}\right)\left(\frac{X_{i}^{(0)}(k)}{a_{i}}+F_{i}\right) \\
+a_{1}\left(\frac{u-b_{1} F_{1}}{a_{1}+b_{1}}\right), \\
X_{1}^{(0)}(k)=\sum_{i=2}^{n} b_{i}^{*}\left(\frac{X_{i}^{(0)}(k)}{a_{i}}+F_{i}\right)+u^{*},
\end{gathered}
$$


where

$$
\begin{aligned}
& b_{i}^{*}=\frac{a_{1} b_{i}}{a_{1}+b_{1}}, \\
& u^{*}=a_{1}\left(\frac{u-b_{1} F_{1}}{a_{1}+b_{1}}\right) .
\end{aligned}
$$

According to the least squares estimation, parameters $b_{2}^{*}, b_{3}^{*}, \ldots, b_{n}^{*}$ and $u^{*}$ of (22) are obtained as

$$
\left[\begin{array}{llllll}
b_{2}^{*} & b_{3}^{*} & b_{4}^{*} & \cdots & b_{n}^{*} & u^{*}
\end{array}\right]^{T}=\left(B^{T} B\right)^{-1} B^{T} Z,
$$

where

$$
\begin{aligned}
& B \\
& =\left[\begin{array}{ccccc}
\frac{X_{2}^{(0)}(1)}{a_{2}}+F_{1} & \frac{X_{3}^{(0)}(1)}{a_{3}}+F_{3} & \cdots & \frac{X_{n}^{(0)}(1)}{a_{n}}+F_{n} & 1 \\
\frac{X_{2}^{(0)}(2)}{a_{2}}+F_{2} & \frac{X_{3}^{(0)}(2)}{a_{3}}+F_{3} & \cdots & \frac{X_{n}^{(0)}(2)}{a_{n}}+F_{n} & 1 \\
\vdots & \vdots & \ddots & \vdots & \vdots \\
\frac{X_{2}^{(0)}(r)}{a_{2}}+F_{2} & \frac{X_{3}^{(0)}(r)}{a_{3}}+F_{3} & \cdots & \frac{X_{n}^{(0)}(r)}{a_{n}}+F_{n} & 1
\end{array}\right], \\
& Z=\left[\begin{array}{c}
X_{1}^{(0)}(1) \\
X_{1}^{(0)}(2) \\
\vdots \\
X_{1}^{(0)}(r)
\end{array}\right] .
\end{aligned}
$$

By solving (2) using the integrating factor method with the initial condition $\widehat{X}_{1}^{(1)}(1)=X_{1}^{(1)}(1)$, the model prediction equation for the predicted 1-AGO series can be obtained as follows.

From (2), the integrating factor is $e^{\int b_{1} d t}=e^{b_{1} t}$. Multiplying (2) by $e^{b_{1} t}$ obtains

$$
\frac{d}{d t}\left(e^{b_{1} t} X_{1}^{(1)}(t)\right)=\sum_{i=2}^{n} e^{b_{1} t} b_{i} X_{i}^{(1)}(t) d t+e^{b_{1} t} u
$$

Taking the integral of both sides of the above equation in the interval $[1, k]$, we get

$$
\begin{aligned}
& \int_{1}^{k} \frac{d}{d t}\left(e^{b_{1} t} X_{1}^{(1)}(t)\right) d t=\int_{1}^{k} \sum_{i=2}^{n} e^{b_{1} t} b_{i} X_{i}^{(1)}(t) d t \\
& \quad+\int_{1}^{k} e^{b_{1} t} u d t, \\
& X_{1}^{(1)}(k) e^{b_{1} k}-X_{1}^{(1)}(1) e^{b_{1}}=\int_{1}^{k} \sum_{i=2}^{n} e^{b_{1} t} b_{i}\left[E_{i} e^{a_{i} t}+F_{i}\right] d t
\end{aligned}
$$

$$
\begin{aligned}
& +\int_{1}^{k} e^{b_{1} t} u d t=\int_{1}^{k} \sum_{i=2}^{n} b_{i}\left[E_{i} e^{\left(b_{1}+a_{i}\right) t}+e^{b_{1} t} F_{i}\right] d t \\
& +\int_{1}^{k} e^{b_{1} t} u d t \\
& X_{1}^{(1)}(k) e^{b_{1} k}=X_{1}^{(1)}(1) e^{b_{1}} \\
& +\sum_{i=2}^{n}\left[b _ { i } \left(\frac{E_{i}\left(e^{\left(b_{1}+a_{i}\right) k}-e^{\left(b_{1}+a_{i}\right)}\right)}{b_{1}+a_{i}}\right.\right. \\
& \left.\left.+\frac{F_{i}\left(e^{b_{1} k}-e^{b_{1}}\right)}{b_{1}}\right)\right]+\frac{u e^{b_{1} k}}{b_{1}}-\frac{u e^{b_{1}}}{b_{1}},
\end{aligned}
$$

Substituting $k=k+1$ into (28) gives

$$
\begin{aligned}
X_{1}^{(1)}(k+1)= & X_{1}^{(1)}(1) e^{-b_{1} k}+\sum_{i=2}^{n} b_{i}\left[\frac{E_{i} e^{a_{i}(k+1)}}{b_{1}+a_{i}}+\frac{F_{i}}{b_{1}}\right] \\
& -\sum_{i=2}^{n} b_{i}\left[\frac{E_{i} e^{-b_{1} k+a_{i}}}{b_{1}+a_{i}}+\frac{F_{i} e^{-b_{1} k}}{b_{1}}\right]+\frac{u}{b_{1}} \\
& -\frac{u e^{-b_{1} k}}{b_{1}} .
\end{aligned}
$$

Multiplying both sides of (28) by $e^{-b_{1}}$, we get

$$
\begin{aligned}
e^{-b_{1}} X_{1}^{(1)}(k)= & X_{1}^{(1)}(1) e^{-b_{1} k} \\
& +\sum_{i=2}^{n} b_{i}\left[\frac{E_{j} e^{a_{i} k-b_{1}}}{b_{1}+a_{i}}+\frac{F_{i} e^{-b_{1}}}{b_{1}}\right] \\
& -\sum_{i=2}^{n} b_{i}\left[\frac{E_{j} e^{-b_{1} k+a_{i}}}{b_{1}+a_{i}}+\frac{F_{i} e^{-b_{1} k}}{b_{1}}\right]+\frac{u e^{-b_{1}}}{b_{1}} \\
& -\frac{u e^{-b_{1} k}}{b_{1}} .
\end{aligned}
$$


Subtracting (30) from (29), we have

$$
\begin{aligned}
& X_{1}^{(1)}(k+1)-e^{-b_{1}} \widehat{X}_{1}^{(1)}(k) \\
& =X_{1}^{(1)}(1) e^{-b_{1} k}+\sum_{i=2}^{n} b_{i}\left[\frac{E_{i} e^{a_{i}(k+1)}}{b_{1}+a_{i}}+\frac{F_{i}}{b_{1}}\right] \\
& -\sum_{i=2}^{n} b_{i}\left[\frac{E_{i} e^{-b_{1} k+a_{i}}}{b_{1}+a_{i}}+\frac{F_{i} e^{-b_{1} k}}{b_{1}}\right]+\frac{u}{b_{1}}-\frac{u e^{-b_{1} k}}{b_{1}} \\
& -X_{1}^{(1)}(1) e^{-b_{1} k}-\sum_{i=2}^{n} b_{i}\left[\frac{E_{i} e^{a_{i} k-b_{1}}}{b_{1}+a_{i}}+\frac{F_{i} e^{-b_{1}}}{b_{1}}\right] \\
& +\sum_{i=2}^{n} b_{i}\left[\frac{E_{i} e^{-b_{1} k+a_{i}}}{b_{1}+a_{i}}+\frac{F_{i} e^{-b_{1} k}}{b_{1}}\right]-\frac{u e^{-b_{1}}}{b_{1}}+\frac{u e^{-b_{1} k}}{b_{1}} \\
& =\sum_{i=2}^{n} b_{i}\left[\frac{E_{i} e^{a_{i}(k+1)}}{b_{1}+a_{i}}+\frac{F_{i}}{b_{1}}\right] \\
& -\sum_{i=2}^{n} b_{i}\left[\frac{E_{i} e^{a_{i} k-b_{1}}}{b_{1}+a_{i}}+\frac{F_{i} e^{-b_{1}}}{b_{1}}\right]+\frac{u}{b_{1}}-\frac{u e^{-b_{1}}}{b_{1}} \\
& =\sum_{i=2}^{n} b_{i}\left[\frac{E_{i} e^{a_{i}(k+1)}+F_{i}}{b_{1}+a_{i}}-\frac{F_{i}}{b_{1}+a_{i}}+\frac{F_{i}}{b_{1}}\right] \\
& -\sum_{i=2}^{n} b_{i}\left[\frac{E_{i} e^{a_{i} k-b_{1}}+F_{i}}{b_{1}+a_{i}}-\frac{F_{i}}{b_{1}+a_{i}}+\frac{F_{i} e^{-b_{1}}}{b_{1}}\right]+\frac{u}{b_{1}} \\
& -\frac{u e^{-b_{1}}}{b_{1}} \\
& =\sum_{i=2}^{n} b_{i}\left[\frac{X_{i}^{(1)}(k+1)}{b_{1}+a_{i}}+\frac{F_{i}}{b_{1}}\right] \\
& -\sum_{i=2}^{n} b_{i}\left[\frac{X_{i}^{(1)}(k) e^{-b_{1}}}{b_{1}+a_{i}}+\frac{F_{i} e^{-b_{1}}}{b_{1}}\right]+\frac{u}{b_{1}}-\frac{u e^{-b_{1}}}{b_{1}} \\
& =\sum_{i=2}^{n} b_{i}\left[\frac{X_{i}^{(1)}(k+1)}{b_{1}+a_{i}}\right]-\sum_{i=2}^{n} b_{i}\left[\frac{X_{i}^{(1)}(k) e^{-b_{1}}}{b_{1}+a_{i}}\right] \\
& +\sum_{i=2}^{n} b_{i}\left[\frac{F_{i}}{b_{1}}-\frac{F_{i} e^{-b_{1}}}{b_{1}}\right]+\frac{u}{b_{1}}-\frac{u e^{-b_{1}}}{b_{1}} \\
& =e^{-b_{1}} \widehat{X}_{1}^{(1)}(k)+\sum_{i=2}^{n} b_{i}\left[\frac{X_{i}^{(1)}(k+1)}{b_{1}+a_{i}}\right] \\
& -\sum_{i=2}^{n} b_{i}\left[\frac{X_{i}^{(1)}(k) e^{-b_{1}}}{b_{1}+a_{i}}\right]+\sum_{i=2}^{n} b_{i}\left[\frac{F_{i}}{b_{1}}-\frac{F_{i} e^{-b_{1}}}{b_{1}}\right] \\
& +\frac{u}{b_{1}}-\frac{u e^{-b_{1}}}{b_{1}} \text {. }
\end{aligned}
$$

Consequently, the predicted 1-AGO series of $X_{1}^{(1)}(k), k=$ $2,3, \ldots, r f$, is given by

$$
\begin{aligned}
\widehat{X}_{1}^{(1)}(k+1)= & c_{1} \widehat{X}_{1}^{(1)}(k)+\sum_{i=2}^{n} c_{i} X_{i}^{(1)}(k+1) \\
& +\sum_{i=2}^{n} d_{i} X_{i}^{(1)}(k)+v \\
\widehat{X}_{1}^{(0)}(k+1)= & \widehat{X}_{1}^{(1)}(k+1)-\widehat{X}_{1}^{(1)}(k)
\end{aligned}
$$

where $c_{1}=e^{-b_{1}}, c_{i}=b_{i} /\left(b_{1}+a_{i}\right), d_{i}=b_{i} c_{1} /\left(b_{1}+a_{i}\right)$, and $v=\sum_{i=2}^{n} b_{i}\left[F_{i} / b_{1}-F_{i} c_{1} / b_{1}\right]+u / b_{1}-u c_{1} / b_{1}$ for $i=2,3, \ldots, n$.

In the traditional grey model, the parameters are evaluated by (23), whereas the model predictions are given by (32). However, a paradox between these two equations would lead to high levels of error of prediction [5]. We can infer that (23) for parameter estimation is equivalent to (32) for model prediction if parameter $b_{1}$ of (2) is zero, which is shown as follows.

Setting $b_{1}=0$ and taking the integral of both sides of (2) in the interval $[1, k]$, we have

$$
\begin{aligned}
& \int_{1}^{k} \frac{d}{d t}\left(X_{1}^{(1)}(t)\right) d t=\int_{1}^{k} \sum_{i=2}^{n} b_{i} X_{i}^{(1)}(t) d t+\int_{1}^{k} u d t \\
& X_{1}^{(1)}(k)-X_{1}^{(1)}(1)=\sum_{i=2}^{n} b_{i} \int_{1}^{k}\left[E_{i} e^{a_{i} t}+F_{i}\right] d t+\int_{1}^{k} u d t \\
&=\sum_{i=2}^{n} b_{i}\left[\frac{E_{i}}{a_{i}}\left(e^{a_{i} k}-e^{a_{i}}\right)+F_{i}(k-1)\right]+u(k-1) \\
&=\sum_{i=2}^{n} b_{i}\left[\frac{X_{i}^{(1)}(k)-X_{i}^{(1)}(1)}{a_{i}}+F_{i}(k-1)\right] \\
& \quad+u(k-1) \\
&=\sum_{i=2}^{n} \frac{b_{i}}{a_{i}}\left(X_{i}^{(1)}(k)-X_{i}^{(1)}(1)\right)+\sum_{i=2}^{n} b_{i} F_{i}(k-1) \\
& \quad+u(k-1), \\
& X_{1}^{(1)}(k-1)-X_{1}^{(1)}(1) \\
&= \sum_{i=2}^{n} \frac{b_{i}}{a_{i}}\left(X_{i}^{(1)}(k-1)-X_{i}^{(1)}(1)\right)+\sum_{i=2}^{n} b_{i} F_{i}(k-2) \\
&+u(k-2) .
\end{aligned}
$$

Substituting $k=k+1$ into (35) gives

$$
\begin{aligned}
X_{1}^{(1)}(k)-X_{1}^{(1)}(1)= & \sum_{i=2}^{n} \frac{b_{i}}{a_{i}}\left(X_{i}^{(1)}(k)-X_{i}^{(1)}(1)\right) \\
& +\sum_{i=2}^{n} b_{i} F_{i}(k-1)+u(k-1) .
\end{aligned}
$$


Subtracting (36) from (35), we have

$$
\begin{aligned}
& X_{1}^{(1)}(k)-X_{1}^{(1)}(k-1) \\
& =\sum_{i=2}^{n} \frac{b_{i}}{a_{i}}\left(X_{i}^{(1)}(k)-X_{i}^{(1)}(k-1)\right)+\sum_{i=2}^{n} b_{i} F_{i}+u, \\
& X_{1}^{(0)}(k)=\sum_{i=2}^{n} b_{i}\left(\frac{X_{i}^{(0)}(k)}{a_{i}}+F\right)+u .
\end{aligned}
$$

Therefore, (32) is the same as (23).

$$
\begin{aligned}
& D=\left[\begin{array}{cccccccccc}
X_{1}^{(1)}(1) & X_{2}^{(1)}(2) & X_{3}^{(1)}(2) & \cdots & X_{n}^{(1)}(2) & X_{2}^{(1)}(1) & X_{3}^{(1)}(1) & \cdots & X_{n}^{(1)}(1) & 1 \\
X_{1}^{(1)}(2) & X_{2}^{(1)}(3) & X_{3}^{(1)}(3) & \cdots & X_{n}^{(1)}(3) & X_{2}^{(1)}(2) & X_{3}^{(1)}(2) & \cdots & X_{n}^{(1)}(2) & 1 \\
\vdots & \vdots & \vdots & \ddots & \vdots & \vdots & \vdots & \ddots & \vdots & \vdots \\
X_{1}^{(1)}(r-1) & X_{2}^{(1)}(r) & X_{3}^{(1)}(r) & \cdots & X_{n}^{(1)}(r) & X_{2}^{(1)}(r-1) & X_{3}^{(1)}(r-1) & \cdots & X_{n}^{(1)}(r-1) & 1
\end{array}\right], \\
& P=\left[\begin{array}{c}
X_{1}^{(1)}(2) \\
X_{1}^{(1)}(3) \\
\vdots \\
X_{1}^{(1)}(r)
\end{array}\right] \\
& c=\left[\begin{array}{llllllllll}
c_{1} & c_{2} & c_{3} & \cdots & c_{n} & d_{2} & d_{3} & \cdots & d_{n} & v
\end{array}\right]^{T} \text {. }
\end{aligned}
$$

The parameters $c_{1}, c_{2}, c_{3}, \ldots, c_{n}, d_{2}, d_{3}, \ldots, d_{n}$ and $v$ are determined using the least squares estimation, as follows.

If $D^{T} D$ is a nonsingular matrix, then the solution of (38) can be obtained using the following equation:

$$
\begin{aligned}
c & =\left[\begin{array}{llllllllll}
c_{1} & c_{2} & c_{3} & \cdots & c_{n} & d_{2} & d_{3} & \cdots & d_{n} & v
\end{array}\right]^{T} \\
& =\left(D^{T} D\right)^{-1} D^{T} P .
\end{aligned}
$$

However, if $D^{T} D$ is a singular matrix, then the solution of (28) can be determined using the equation

$$
c=\left[\begin{array}{llllllllll}
c_{1} & c_{2} & c_{3} & \cdots & c_{n} & d_{2} & d_{3} & \cdots & d_{n} & v
\end{array}\right]^{T}=D^{+} P,
$$

where $D^{+}$is Moore-Penrose pseudoinverse of matrix $D$ [25, 26].

Finally, the predicted series of $X_{1}^{(0)}(k), k=2,3, \ldots, r f$ is calculated by using (32) and (9).

\section{Statistical Measure of the Forecasting Performance}

To evaluate the performance of model simulation and prediction, two criteria, namely, the mean absolute percentage error (MAPE) and the root mean square percentage error (RMSPE), are applied for this study. MAPE and RMSPE are the most commonly used accuracy measures in prediction
The different forms of these two equations affect the prediction accuracy of the model. Therefore, in this study, (32) is used for parameter estimation and model prediction in order to overcome this problem.

Firstly, we substitute all the data values into (32). Then, the linear system is derived as follows:

$$
D c=P \text {, }
$$

where

models [27-30]. Generally, MAPE and RMSPE are defined, respectively, as

$$
\begin{aligned}
\text { MAPE }= & \frac{1}{r-1} \sum_{k=2}^{r}\left|\frac{X^{(0)}(k)-\widehat{X}^{(0)}(k)}{X^{(0)}(k)}\right| \times 100 \%, \\
\text { RMSPE }= & \sqrt{\frac{\sum_{k=2}^{r}\left(\left(X^{(0)}(k)-\widehat{X}^{(0)}(k)\right) / X^{(0)}(k)\right)^{2}}{r-1}} \\
& \times 100 \%,
\end{aligned}
$$

where $X^{(0)}(k)$ is the actual value at time $k, \widehat{X}^{(0)}(k)$ is its model value, and $r$ is the number of data used for prediction. The values of MAPE and RMSPE sufficiently describe the goodness of prediction effect. The lower the values of MAPE and RMSPE, the more accurate the prediction. The criteria of MAPE and RMSPE are presented in Table $1[29,30]$. In addition, absolute percentage error (APE) is used to evaluate the accuracy of the model for each data point, which is defined as

$$
\mathrm{APE}=\left|\frac{X^{(0)}-\widehat{X}^{(0)}}{X^{(0)}}\right| \times 100 \% .
$$


TABLE 1: Criteria of MAPE and RMSPE.

\begin{tabular}{lc}
\hline MAPE and RMSPE (\%) & Forecasting power \\
\hline$<10$ & Highly accurate forecasting \\
$10-20$ & Good forecasting \\
$20-50$ & Reasonable forecasting \\
$>50$ & Inaccurate forecasting \\
\hline
\end{tabular}

\section{Application of Modified GMC $(1, n)$ Model}

In order to verify the performance of the modified $\operatorname{GMC}(1, n)$ prediction model, two different real cases of prediction problems are considered. In addition, we compare prediction accuracy with the conventional $\operatorname{GMC}(1, n)$ and the discrete multivariate grey model (D-GMC $(1, n))$.

Case 1 (forecasting $\mathrm{CO}_{2}$ emission levels in Thailand). From a previous study [31], it has been established that the main factors that affect the amount of $\mathrm{CO}_{2}$ released into the atmosphere are natural gas, solid fuel, liquid fuel, population, and GDP. Therefore, these factors are used to predict the $\mathrm{CO}_{2}$ emission levels in Thailand for this study. All the data, from 1994 to 2013, were obtained from the Energy Policy and Planning Office (EPPO) of Thailand (data source: http://www.eppo.go.th/info/index-statistics.html).

These factors along with the factor of $\mathrm{CO}_{2}$ emission are defined as the variables in the modified grey model $\operatorname{GMC}(1,6)$, which are written as follows:

$X_{1}^{(0)}(t)$ is the time series of $\mathrm{CO}_{2}$ emission (1,000 tons).

$X_{2}^{(0)}(t)$ is the time series of natural gas consumption (MMSCFD).

$X_{3}^{(0)}(t)$ is the time series of solid fuel consumption (1,000 tons).

$X_{4}^{(0)}(t)$ is the time series of liquid fuel consumption (barrels/day).

$X_{5}^{(0)}(t)$ is the time series of population (persons).

$X_{6}^{(0)}(t)$ is the time series of GDP (billion bahts).

$t$ is an order of the time series: $t=1$ refers to the year 1994 and $t=20$ refers to the year 2013 .

The actual data are separated into two parts: the first part (1994-2010) is used to construct the models, while the second part (2011-2013) is employed for model prediction. All the data satisfy the quasi-smooth and quasi-exponential conditions. The result of the modified $\operatorname{GMC}(1,6)$ model, obtained from (32), has the form

$$
\begin{aligned}
\widehat{X}_{1}^{(1)}(k+1)= & 0.248 \widehat{X}_{1}^{(1)}(k)-1.133 X_{2}^{(1)}(k+1) \\
& -1.359 X_{2}^{(1)}(k)+0.326 X_{3}^{(1)}(k+1)
\end{aligned}
$$

$$
\begin{aligned}
& +0.227 X_{3}^{(1)}(k)+0.487 X_{4}^{(1)}(k+1) \\
& +0.300 X_{4}^{(1)}(k)+0.031 X_{5}^{(1)}(k+1) \\
& +0.015 X_{5}^{(1)}(k)+0.064 X_{6}^{(1)}(k+1) \\
& -0.095 X_{6}^{(1)}(k)+1.285, \\
& k=1,2, \ldots, 19 .
\end{aligned}
$$

The actual values, the model values, the predictive values, and the APE of D-GMC $(1,6)$ and the modified $\operatorname{GMC}(1,6)$ models are presented in Table 2 . Table 3 shows the performance of the model simulation and prediction of D-GMC $(1,6)$ as well as the modified $\operatorname{GMC}(1,6)$. As demonstrated in these tables, both the models have highly accurate forecasting abilities. However, the modified $\operatorname{GMC}(1,6)$ has lower MAPE and RMSPE than D-GMC $(1,6)$, which indicates that the modified $\operatorname{GMC}(1,6)$ has higher accuracy of prediction than $\mathrm{D}-\operatorname{GMC}(1,6)$. As far as the conventional $\operatorname{GMC}(1,6)$ is concerned, large errors were obtained for the model simulation and prediction (results not shown).

Case 2 (forecasting electricity consumption in Thailand). From a previous study [32], it has been established that population, gross domestic product (GDP), stock index (SET index), and total revenue from exporting industrial products (export) are the main factors that influence the electricity consumption (measured in GWh) of Thailand. Therefore, we used these factors to construct the grey model. All data were obtained from the Energy Policy and Planning Office and Stock Exchange of Thailand. The data set was collected annually from 2002 to 2014 and is as presented in Table 4. These factors and electricity consumption are defined as the variables in the modified grey model $\operatorname{GMC}(1,5)$, which are written as follows:

$X_{1}^{(0)}(t)$ is the time series of electricity consumption (GWh).

$X_{2}^{(0)}(t)$ is the time series of population (persons).

$X_{3}^{(0)}(t)$ is the time series of gross domestic product (billion bahts).

$X_{4}^{(0)}(t)$ is the time series of stock index (point).

$X_{5}^{(0)}(t)$ is the time series of the total revenue from exporting industrial products (billion bahts).

$t$ is an order of the time series: $t=1$ refers to the year 2002 and $t=13$ refers to the year 2014 .

We will use the steps of the modified grey $\operatorname{GMC}(1,5)$ model for finding the parameters from 2002 to 2011 for the prediction of electricity consumption (measured in GWh) from 2012 to 2014 . All the data satisfy the quasi-smooth 
TABLE 2: Comparison of actual values, model values, and APEs of D-GMC $(1,6)$ and modified GMC $(1,6)$ models.

\begin{tabular}{|c|c|c|c|c|c|c|}
\hline \multirow{2}{*}{ Years } & \multirow{2}{*}{$t$} & \multirow{2}{*}{$\mathrm{CO}_{2}$ emission data } & \multicolumn{2}{|c|}{ D-GMC $(1,6)$} & \multicolumn{2}{|c|}{ Modified GMC $(1,6)$} \\
\hline & & & Model value & APE & Model value & APE \\
\hline 1994 & 1 & $122,876.13$ & $122,876.13$ & 0.00 & $122,876.13$ & 0.00 \\
\hline 1995 & 2 & $137,691.67$ & $142,056.72$ & 3.17 & $138,089.13$ & 0.29 \\
\hline 1996 & 3 & $155,553.15$ & $148,750.34$ & 4.37 & $154,325.20$ & 0.79 \\
\hline 1997 & 4 & $161,057.54$ & $154,933.62$ & 3.80 & $162,115.22$ & 0.66 \\
\hline 1998 & 5 & $145,451.52$ & $161,292.65$ & 10.89 & $146,188.49$ & 0.51 \\
\hline 1999 & 6 & $149,881.53$ & $140,012.17$ & 6.58 & $148,098.26$ & 1.19 \\
\hline 2000 & 7 & $150,832.00$ & $150,968.83$ & 0.09 & $150,578.46$ & 0.17 \\
\hline 2001 & 8 & $153,545.22$ & $157,968.41$ & 2.88 & $156,157.34$ & 1.70 \\
\hline 2002 & 9 & $163,558.08$ & $164,645.66$ & 0.66 & $161,506.52$ & 1.25 \\
\hline 2003 & 10 & $172,053.83$ & $176,232.82$ & 2.43 & $172,557.16$ & 0.29 \\
\hline 2004 & 11 & $187,418.22$ & $176,180.65$ & 6.00 & $189,201.76$ & 0.95 \\
\hline 2005 & 12 & $192,574.90$ & $193,410.05$ & 0.43 & $188,058.52$ & 2.35 \\
\hline 2006 & 13 & $193,233.99$ & $197,621.97$ & 2.27 & $196,939.39$ & 1.92 \\
\hline 2007 & 14 & $200,494.35$ & $194,241.16$ & 3.12 & $199,340.27$ & 0.58 \\
\hline 2008 & 15 & $203,261.89$ & $208,243.34$ & 2.45 & $204,008.47$ & 0.37 \\
\hline 2009 & 16 & $208,267.86$ & $211,387.03$ & 1.50 & $207,577.79$ & 0.33 \\
\hline 2010 & 17 & $220,467.16$ & $217,488.33$ & 1.35 & $220,488.97$ & 0.01 \\
\hline $2011^{*}$ & 18 & $224,465.07$ & $236,377.49$ & 5.31 & $222,859.77$ & 0.72 \\
\hline $2012^{*}$ & 19 & $240,583.97$ & $234,631.65$ & 2.47 & $240,026.56$ & 0.23 \\
\hline $2013^{*}$ & 20 & $240,802.07$ & $248,902.18$ & 3.36 & $241,530.87$ & 0.30 \\
\hline
\end{tabular}

${ }^{*}$ Prediction.

TABLe 3: Performance evaluation for fitting and Prediction accuracies of D-GMC $(1,6)$ and modified GMC $(1,6)$ models.

\begin{tabular}{|c|c|c|c|c|}
\hline \multirow{2}{*}{ Criteria } & \multicolumn{2}{|c|}{ D-GMC $(1,6)$} & \multicolumn{2}{|c|}{ Modified GMC $(1,6)$} \\
\hline & Model value & Predictive value & Model value & Predictive value \\
\hline MAPE (\%) & 3.25 & 3.71 & 0.83 & 0.42 \\
\hline RMSPE (\%) & 4.19 & 3.90 & 1.06 & 0.47 \\
\hline
\end{tabular}

and quasi-exponential conditions. The result of the modified GMC $(1,5)$ model, obtained from $(32)$, has the form

$$
\begin{aligned}
& \widehat{X}_{1}^{(1)}(k+1)= 0.417 \widehat{X}_{1}^{(1)}(k)+0.398 X_{2}^{(1)}(k+1) \\
&-0.025 X_{2}^{(1)}(k)+0.910 X_{3}^{(1)}(k+1) \\
&-0.491 X_{3}^{(1)}(k)+0.006 X_{4}^{(1)}(k+1) \\
&-0.013 X_{4}^{(1)}(k)-0.139 X_{5}^{(1)}(k+1) \\
&-0.018 X_{5}^{(1)}(k)-0.226, \\
& k=1,2, \ldots, 12 .
\end{aligned}
$$

The actual values, the model values, the predictive values, and the APE of D-GMC $(1,5)$ and the modified $\operatorname{GMC}(1,5)$ models are presented in Table 4 . Table 5 shows the MAPE and RMSPE values of D-GMC $(1,5)$ and the modified $\operatorname{GMC}(1,5)$ models, which are used to measure the accuracy of prediction of the models. These results indicate that the modified GMC $(1,5)$ has lower MAPE and RMSPE values than D$\operatorname{GMC}(1,5)$. For the conventional $\operatorname{GMC}(1,5)$, large errors were obtained for the model simulation and prediction (results not shown). Therefore, it can be safely concluded that the modified $\operatorname{GMC}(1,5)$ has a higher degree of accuracy of prediction than the conventional $\operatorname{GMC}(1,5)$ and $\mathrm{D}$ $\operatorname{GMC}(1,5)$.

\section{Conclusion}

This study presents a modified $\operatorname{GMC}(1, n)$ model for improving the prediction accuracy of conventional $\operatorname{GMC}(1, n)$ by modification of the formula for calculating the background value, the system of parameter estimation, and the model prediction equation. The prediction accuracy can be compared between the conventional $\operatorname{GMC}(1, n)$, D-GMC $(1, n)$, and the modified $\operatorname{GMC}(1, n)$ models in terms of the MAPE and RMSPE values. The empirical results obtained using the modified $\operatorname{GMC}(1, n)$ reveal the least error for MAPE and RMSPE in comparison with conventional $\operatorname{GMC}(1, n)$ and D$\operatorname{GMC}(1, n)$ for two real cases of prediction problems. This success indicates that the modified $\operatorname{GMC}(1, n)$ improves the accuracy of the simulation and prediction of the conventional $\mathrm{GMC}(1, n)$ models. In addition, using the criteria in Table 1 , it can be substantiated that the modified $\operatorname{GMC}(1, n)$ 
TABLE 4: Comparison of actual values, model values, and APE of D-GMC $(1,5)$ and modified GMC $(1,5)$ models.

\begin{tabular}{|c|c|c|c|c|c|c|}
\hline \multirow{2}{*}{ Years } & \multirow{2}{*}{$t$} & \multirow{2}{*}{ Electricity consumption data } & \multicolumn{2}{|c|}{ D-GMC $(1,5)$} & \multicolumn{2}{|c|}{ Modified GMC $(1,5)$} \\
\hline & & & Model value & APE & Model value & APE \\
\hline 2002 & 1 & 100,091 & $100,091.00$ & 0.000 & 100091.00 & 0 \\
\hline 2003 & 2 & 106,987 & $105,968.82$ & 0.952 & 106987.17 & $4.11 \times 10^{-12}$ \\
\hline 2004 & 3 & 115,101 & $117,863.18$ & 2.400 & 115101.15 & $1.29 \times 10^{-11}$ \\
\hline 2005 & 4 & 121,240 & $119,605.49$ & 1.348 & 121240.03 & $1.28 \times 10^{-11}$ \\
\hline 2006 & 5 & 127,879 & $127,053.81$ & 0.645 & 127879.26 & $1.25 \times 10^{-11}$ \\
\hline 2007 & 6 & 133,113 & $132,870.66$ & 0.182 & 133112.82 & $1.24 \times 10^{-11}$ \\
\hline 2008 & 7 & 135,520 & $137,355.47$ & 1.354 & 135520.31 & $1.24 \times 10^{-11}$ \\
\hline 2009 & 8 & 135,181 & $134,748.54$ & 0.320 & 135180.80 & $1.22 \times 10^{-11}$ \\
\hline 2010 & 9 & 149,301 & $148,048.60$ & 0.839 & 149301.14 & $1.15 \times 10^{-11}$ \\
\hline 2011 & 10 & 148,855 & $149,991.01$ & 0.763 & 148855.54 & $1.28 \times 10^{-11}$ \\
\hline $2012^{*}$ & 11 & 161,779 & $169,923.11$ & 5.034 & 159807.95 & 1.22 \\
\hline $2013^{*}$ & 12 & 164,341 & $161,959.15$ & 1.449 & 164233.19 & 0.07 \\
\hline $2014^{*}$ & 13 & 168,620 & $165,305.30$ & 1.966 & 163396.00 & 3.10 \\
\hline
\end{tabular}

${ }^{*}$ Prediction.

TABLE 5: Performance evaluation for fitting and prediction accuracies of D-GMC $(1,5)$, and modified GMC $(1,5)$ models.

\begin{tabular}{lcccc}
\hline \multirow{2}{*}{ Error } & \multicolumn{2}{c}{ D-GMC(1,5) } & \multicolumn{2}{c}{ Modified GMC $(1,5)$} \\
& Model value & Predictive value & Model value & Predictive value \\
\hline MAPE (\%) & 0.98 & 2.82 & $1.15 \times 10^{-11}$ & 1.46 \\
RMSPE (\%) & 1.16 & 3.23 & $1.17 \times 10^{-11}$ & 1.92 \\
\hline
\end{tabular}

model demonstrates highly accurate forecasting. However, the modified $\operatorname{GMC}(1, n)$ model needs to be validated with more real problems and cannot be directly written in the form of the differential equation which is given as (2). For practical applications, the proposed model can be applied to many real systems, especially the systems which have the similarity in trend between the predicted variables and their influencing factors [8]. In addition, the proposed techniques can be applied to the real studies $[10,15,21,22]$ which have successfully used the conventional $\operatorname{GMC}(1, n)$.

\section{Conflict of Interests}

The authors declare that there is no conflict of interests regarding the publication of this paper.

\section{Acknowledgment}

This research was supported by Chiang Mai University.

\section{References}

[1] J. L. Deng, "Control problem of grey systems," Systems and Control Letters, vol. 1, no. 5, pp. 288-294, 1982.

[2] T. Leephakpreeda, "Grey prediction on indoor comfort temperature for HVAC systems," Expert Systems with Applications, vol. 34, no. 4, pp. 2284-2289, 2008.
[3] Y. M. Atwa and E. F. El-Saadany, "Annual wind speed estimation utilizing constrained grey predictor," IEEE Transactions on Energy Conversion, vol. 24, no. 2, pp. 548-550, 2009.

[4] L. Youxin, W. Xiao, L. Min, and C. Anhui, "Grey dynamic model $\mathrm{GM}(1, \mathrm{~N})$ for the relationship of cost and variability," Kybernetes, vol. 38, no. 3-4, pp. 435-440, 2009.

[5] Z. He, Q. Wang, Y. Shen, and Y. Wang, "Discrete multivariate gray model based boundary extension for bi-dimensional empirical mode decomposition," Signal Processing, vol. 93, no. 1, pp. 124-138, 2013.

[6] Z.-X. Wang and L.-L. Pei, "An optimized Grey dynamic model for forecasting the output of high-tech industry in China," Mathematical Problems in Engineering, vol. 2014, Article ID 586284, 7 pages, 2014.

[7] L. Liu, Q. Wang, M. Liu, and L. Li, "An intelligence optimized rolling grey forecasting model fitting to small economic dataset," Abstract and Applied Analysis, vol. 2014, Article ID 641514, 10 pages, 2014.

[8] Z.X. Wang, "Nonlinear grey prediction model with convolution integral NGMC $(1, n)$ and its application to the forecasting of China's industrial $\mathrm{SO}_{2}$ emissions," Journal of Applied Mathematics, vol. 2014, Article ID 580161, 9 pages, 2014.

[9] S. Liu and Y. Lin, Grey Information-Theory, and Practical Applications, Springer, London, UK, 2006.

[10] T.-L. Tien, "The indirect measurement of tensile strength of material by the grey prediction model $\operatorname{GMC}(1, n)$," Measurement Science and Technology, vol. 16, no. 6, pp. 1322-1328, 2005.

[11] T. Y. Pai, R. J. Chiou, and H. H. Wen, "Evaluating impact level of different factors in environmental impact assessment for 
incinerator plants using GM $(1, N)$ model," Waste Management, vol. 28, no. 10, pp. 1915-1922, 2008.

[12] L.-C. Hsu, "Forecasting the output of integrated circuit industry using genetic algorithm based multivariable grey optimization models," Expert Systems with Applications, vol. 36, no. 4, pp. 7898-7903, 2009.

[13] L.-C. Hsu and C.-H. Wang, "Forecasting integrated circuit output using multivariate grey model and grey relational analysis," Expert Systems with Applications, vol. 36, no. 2, pp. 1403-1409, 2009.

[14] T.-L. Tien, "A research on the grey prediction model GM( $1, n)$," Applied Mathematics and Computation, vol. 218, no. 9, pp. 49034916, 2012.

[15] W.-Y. Wu and S.-P. Chen, "A prediction method using the grey model GMC $(1, n)$ combined with the grey relational analysis: a case study on internet access population forecast," Applied Mathematics and Computation, vol. 169, no. 1, pp. 198-217, 2005.

[16] T.-L. Tien, "The deterministic grey dynamic model with convolution integral DGDMC $(1, n)$," Applied Mathematical Modelling, vol. 33, no. 8, pp. 3498-3510, 2009.

[17] H. Guo, X. Xiao, and J. Forrest, "A research on a comprehensive adaptive grey prediction model CAGM $(1, N)$," Applied Mathematics and Computation, vol. 225, pp. 216-227, 2013.

[18] M. Guo, J.-H. Lan, and J.-J. Li, "Traffic flow data recovery algorithm based on gray residual GM(1,N) model," Journal of Transportation Systems Engineering and Information Technology, vol. 12, no. 1, pp. 42-47, 2012.

[19] J. Ren, S. Gao, S. Tan, and L. Dong, "Prediction of the yield of biohydrogen under scanty data conditions based on $\operatorname{GM}(1, N)$," International Journal of Hydrogen Energy, vol. 38, no. 30, pp. 13198-13203, 2013.

[20] R. Guo, "Modeling imperfectly repaired system data via grey differential equations with unequal-gapped times," Reliability Engineering \& System Safety, vol. 92, no. 3, pp. 378-391, 2007.

[21] S. P. Chen and C. M. Shih, "Diffusion forecasting of innovative products using an improved grey model," The Journal of Grey System, vol. 10, no. 1, pp. 23-32, 2007.

[22] K. Peng and X. Xiao, "GMC $(1, N)$ power model based on cluster analysis and its application in predicting ultimate bearing capacity of single pile," in Proceedings of the 2nd International Conference on Information Engineering and Computer Science (ICIECS '10), pp. 1-4, Wuhan, China, December 2010.

[23] N.-M. Xie and S.-F. Liu, "Discrete grey forecasting model and its optimization," Applied Mathematical Modelling, vol. 33, no. 2, pp. 1173-1186, 2009.

[24] L. Wu, S. Liu, L. Yao, and S. Yan, “The effect of sample size on the grey system model," Applied Mathematical Modelling, vol. 37, no. 9, pp. 6577-6583, 2013.

[25] N. R. Farnum and L. W. Stanton, Quantitative Forecasting Method, PWS-Kent, Boston, Mass, USA, 1989.

[26] Y. Q. Zhang, Y. Ai, K. J. Dai, and G. D. Zhang, "Grey model via polynomial for image denoising," Journal of Grey System, vol. 22, no. 2, pp. 117-128, 2010.

[27] L.-C. Hsu, "Using improved grey forecasting models to forecast the output of opto-electronics industry," Expert Systems with Applications, vol. 38, no. 11, pp. 13879-13885, 2011.

[28] S.-L. Ou, "Forecasting agricultural output with an improved grey forecasting model based on the genetic algorithm," Computers and Electronics in Agriculture, vol. 85, pp. 33-39, 2012.

[29] C. Lewis, Industrial and Business Forecasting Methods, Butterworth Scientific, London, UK, 1982.
[30] S. A. DeLurgio, Forecasting Principles and Applications, Irwin/ McGraw-Hill, New York, NY, USA, 1998.

[31] V. Bevilacqua, F. Intini, and S. Kühtz, "A model of artificial neural network for the analysis of climate change," in Proceedings of the 28th International Symposium on Forecasting, pp. 22-25, Nice, France, June 2008.

[32] K. Kandananond, "Forecasting electricity demand in Thailand with an artificial neural network approach," Energies, vol. 4, no. 8, pp. 1246-1257, 2011. 

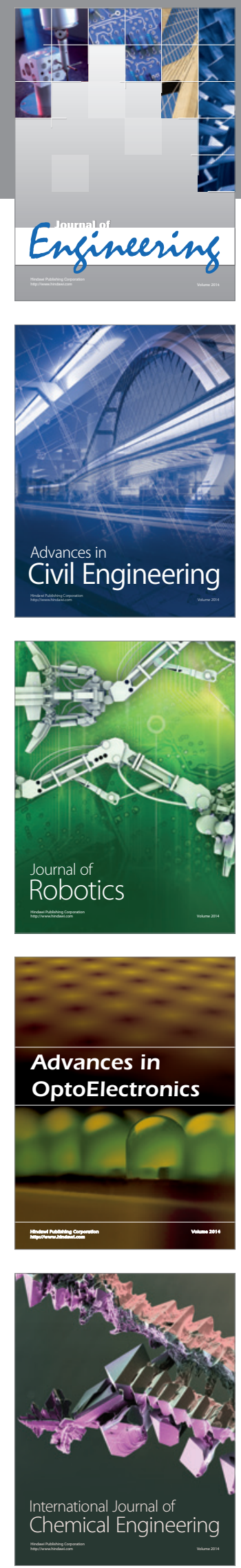

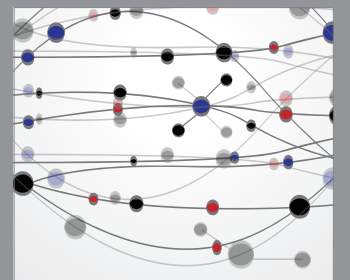

The Scientific World Journal
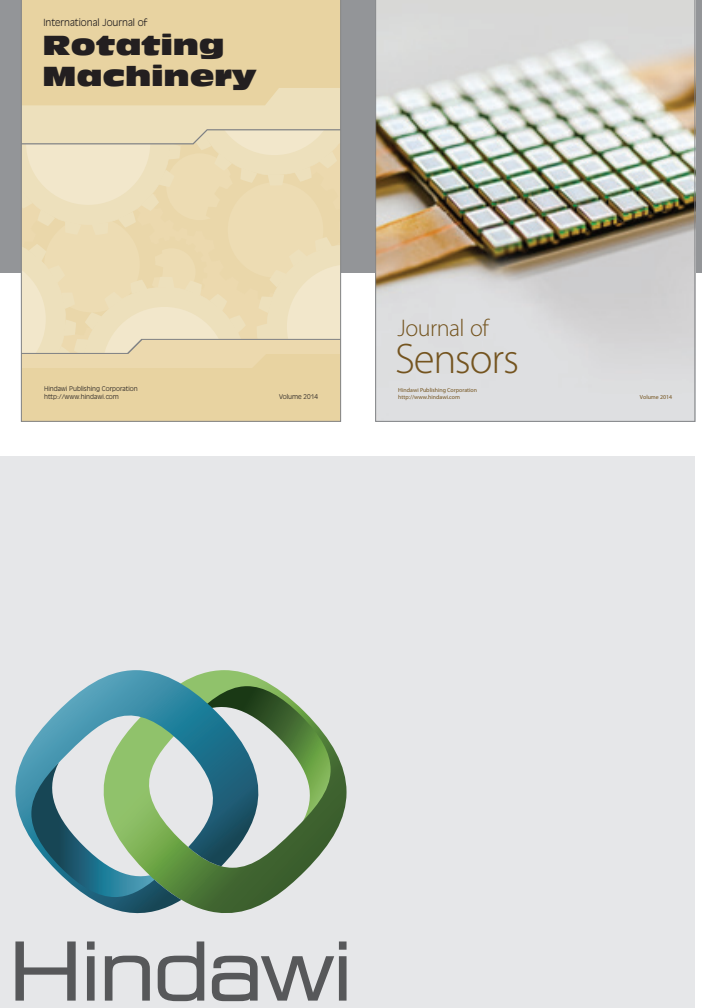

Submit your manuscripts at http://www.hindawi.com
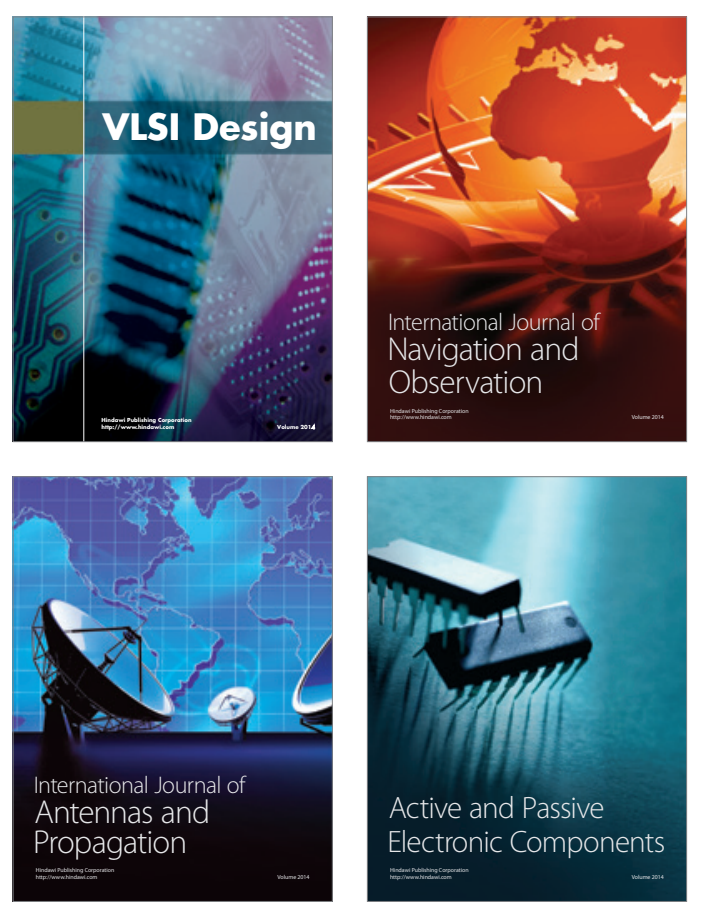
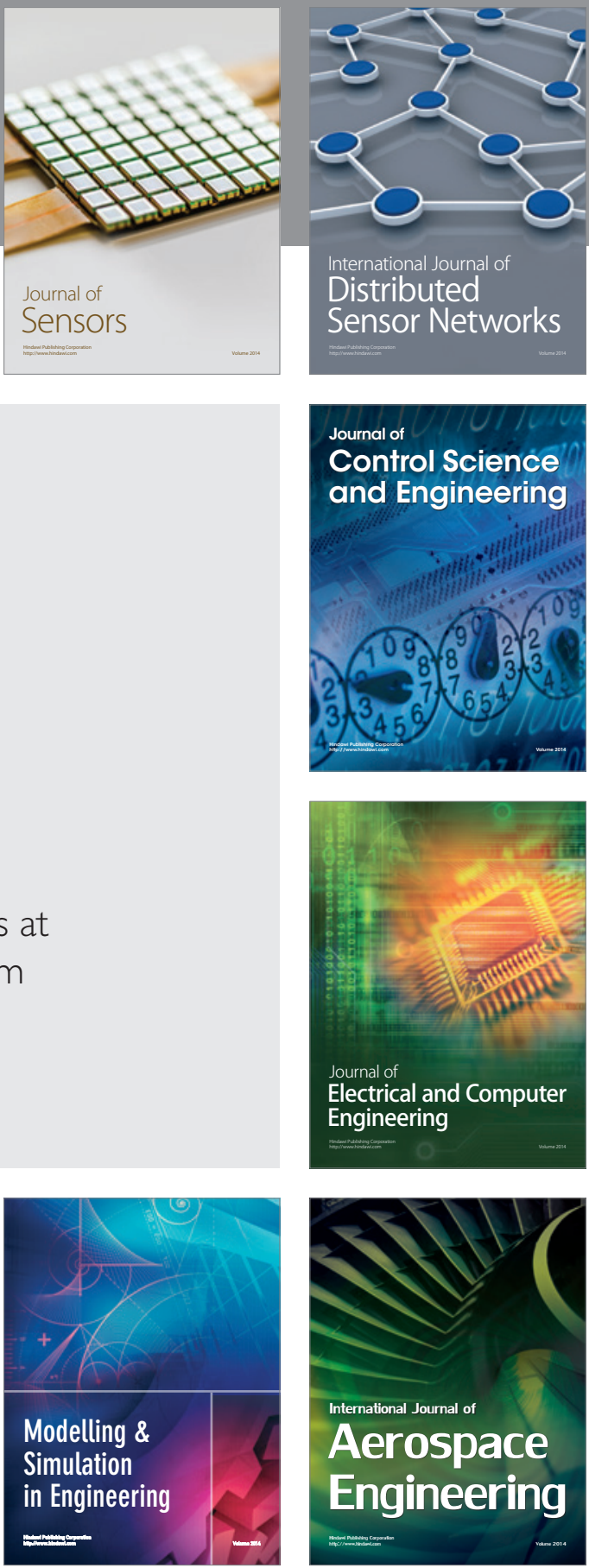

Journal of

Control Science

and Engineering
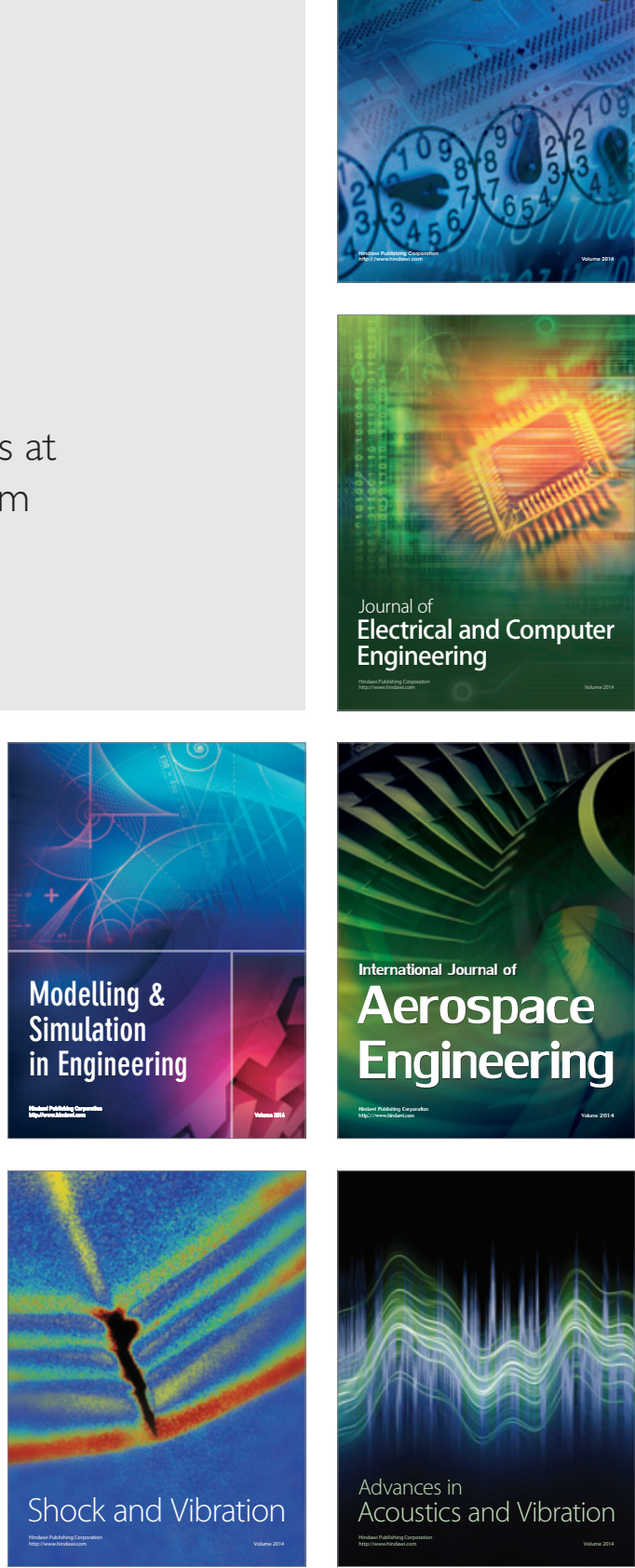